\title{
THE STOCHASTIC ORDER OF PROBABILITY MEASURES ON ORDERED METRIC SPACES
}

\author{
FUMIO HIAI, JIMMIE LAWSON, YONGDO LIM
}

\begin{abstract}
The general notion of a stochastic ordering is that one probability distribution is smaller than a second one if the second attaches more probability to higher values than the first. Motivated by recent work on barycentric maps on spaces of probability measures on ordered Banach spaces, we introduce and study a stochastic order on the space of probability measures $\mathcal{P}(X)$, where $X$ is a metric space equipped with a closed partial order, and derive several useful equivalent versions of the definition. We establish the antisymmetry and closedness of the stochastic order (and hence that it is a closed partial order) for the case of a partial order on a Banach space induced by a closed normal cone with interior. We also consider order-completeness of the stochastic order for a cone of a finite-dimensional Banach space and derive a version of the arithmetic-geometric-harmonic mean inequalities in the setting of the associated probability space on positive matrices.
\end{abstract}

2010 Mathematics Subject Classification. Primary 60B11, 28A33 Secondary 47B65, 28B15, 54E70

Key words and phrases. stochastic order, Borel probability measure, ordered metric spaces, normal cones, Wasserstein metric, AGH mean inequalities.

\section{INTRODUCTION}

The stochastic order for random variables $X, Y$ from a probability measure space $(M, P)$ to $\mathbb{R}$ is defined by $X \leq Y$ if $P(X>t) \leq P(Y>t)$ for all $t \in \mathbb{R}$. This notion extends directly to random variables into $\mathbb{R}^{n}$ equipped with the coordinatewise order. Alternatively one can define a stochastic order on the Borel probability measures on $\mathbb{R}$ or $\mathbb{R}^{n}$ by $\mu \leq \nu$ if for each $s \in \mathbb{R}, \mu(s<t) \leq \nu(s<t)$, where $(s<t):=\{t \in \mathbb{R}: s<t\}$. One then has for random variables $X, Y, X \leq Y$ in the stochastic order if and only if $P_{X} \leq P_{Y}$, where $P_{X}, P_{Y}$ are the push-forward probability measures with respect to $X, Y$ respectively. 
There are important metric spaces which are equipped with a naturally defined partial order, for example the open cone $\mathbb{P}_{n}$ of positive definite matrices of some fixed dimension, where the order is the Loewner order. One can use the Loewner order to define an order on $\mathcal{P}\left(\mathbb{P}_{n}\right)$, the space of Borel probability measures, an order that we call that stochastic order, as it generalizes the case of $\mathbb{R}$ or $\mathbb{R}^{n}$.

In this paper we broadly generalize the stochastic order to an order on the set of Borel probability measures on a partially ordered metric space. We develop basic properties of this order and specialize to the setting of normal cones in Banach spaces to show that the stochastic order in that setting is indeed a partial order.

In Section 3 we give the general definition of the stochastic order on $\mathcal{P}(X)$ for a partially ordered metric space $X$ and derive several useful alternative formulations. In Section 4 we show for normal cones with interior that the stochastic order on $\mathcal{P}(X)$ is indeed a partial order (the antisymmetry being the nontrivial property to establish). In Section 5 we show in the normal cone setting that the stochastic partial order is a closed order with respect to the weak topology, and hence with respect to the Wasserstein topology. In Section 6 we consider the order-completeness of $\mathcal{P}(X)$, and in Section 7 derive a version of the arithmetic-geometric-harmonic means inequality in the setting of the probability space $\mathcal{P}(\mathbb{P})$ on the cone $\mathbb{P}$ of positive invertible operators on a Hilbert space.

In what follows $\mathbb{R}^{+}=[0, \infty)$.

\section{Borel measures}

In this section we recall some basic results about Borel measures on metric spaces that will be needed in what follows. As usual the Borel algebra on a metric space $(X, d)$ is the smallest $\sigma$-algebra containing the open sets and a finite positive Borel measure is a countably additive measure $\mu$ defined on the Borel sets such that $\mu(X)<$ $\infty$. We work exclusively with finite positive Borel measures, primarily those that are probability measures.

Recall that a Borel measure $\mu$ is $\tau$-additive if $\tau(U)=\sup _{\alpha} \tau\left(U_{\alpha}\right)$ for any directed union $U=\bigcup_{\alpha} U_{\alpha}$ of open sets. The measure $\mu$ is said to be inner regular or tight if for any Borel set $A$ and $\varepsilon>0$ there exists a compact set $K \subseteq A$ such that $\mu(A)-\varepsilon<\mu(K)$. A tight finite Borel measure is also called a Radon measure. 
Probability on metric spaces has been carried out primarily for separable metric spaces, although results exist for the non-separable setting. We recall the following result, which can be more-or-less cobbled together from results in the literature; see [7] for more details.

Proposition 2.1. A finite Borel measure $\mu$ on a metric space $(X, d)$ has separable support. The following three conditions are equivalent:

(1) The support of $\mu$ has measure $\mu(X)$.

(2) The measure $\mu$ is $\tau$-additive.

(3) The measure $\mu$ is the weak limit of a sequence of finitely supported measures. If in addition $X$ is complete, these are also equivalent to:

(4) The measure $\mu$ is inner regular.

Proof. For a proof of separability and the equivalence of the first three conditions, see [7]. Suppose (1)-(3) hold and $X$ is complete. Let $\mu$ be a finite Borel measure. Then the support $S$ of $\mu$ is closed, separable and has measure 1. Let $A$ be any Borel measurable set. Then $\mu(A \cap(X \backslash S))=0$ since $\mu(X \backslash S)=0$, so $\mu(A)=\mu(A \cap S)$. Since the metric space $S$ is a separable complete metric space, it is a standard result that $\left.\mu\right|_{S}$ is an inner regular measure. Thus for $\varepsilon>0$ there exists a compact set $K \subseteq S \cap A \subseteq A$ such that $\mu(A)=\mu(A \cap S)<\mu(K)+\varepsilon$.

Conversely suppose $\mu$ is inner regular. If $\mu(S)<\mu(X)$ for the support $S$ of $\mu$, then for $U=X \backslash S, \mu(U)>0$. By inner regularity there exists a compact set $K \subseteq U$ such that $\mu(K)>0$. Since $K$ misses the support of $\mu$, for each $x \in K$, there exists an open set $U_{x}$ containing $x$ such that $\mu\left(U_{x}\right)=0$. Finitely many of the $\left\{U_{x}\right\}$ cover $K$, the finite union has measure 0 , so the subset $K$ has measure 0 , a contradiction. So the support of $\mu$ has measure $\mu(X)$.

Remark 2.2. Finite Borel measures on separable metric spaces are easily shown to be $\tau$-additive and hence satisfy the other equivalent conditions of Proposition 2.1. Finite Borel measures that fail to satisfy the previous conclusions are rare. Indeed it is a theorem that in a complete metric space $X$ there exists a finite Borel measure that fails to be inner regular if and only if the minimal cardinality $w(X)$ for a basis of open sets of $X$ is a measurable cardinal; see volume 4, page 244 of [4]. The existence of measurable cardinals is an axiom independent of the basic Zermelo-Fraenkel axioms 
of set theory and thus if its negation is assumed, all finite Borel measures on complete metric spaces satisfy the four conditions of Proposition 2.1.

\section{THE STOCHASTIC ORDER}

We henceforth restrict our attention to the set of Borel probability measures on a metric space $X$ satisfying the four conditions of Proposition 2.1 and denote this set $\mathcal{P}(X)$. For complete separable metric spaces the set $\mathcal{P}(X)$ consists of all Borel probability measures, which are automatically $\tau$-additive in this case.

Definition 3.1. A partially ordered topological space is a space equipped with a closed partial order $\leq$, one for which $\{(x, y): x \leq y\}$ is closed in $X \times X$.

For a nonempty subset $A$ of a partially ordered set $P$, let $\uparrow A:=\{y \in P: \exists x \in$ $A, x \leq y\}$. The set $\downarrow A$ is defined in an order-dual fashion. A set $A$ is an upper set if $\uparrow A=A$ and a lower set if $\downarrow A=A$. We abbreviate $\uparrow\{x\}$ by $\uparrow x$ and $\downarrow\{x\}$ by $\downarrow x$.

Lemma 3.2. A partially ordered topological space is Hausdorff. If $K$ is a nonempty compact subset, then $\uparrow K$ and $\downarrow K$ are closed.

Proof. See Section VI-1 of [5].

The following definition captures in the setting of ordered topological spaces the notion that higher values should have higher probability.

Definition 3.3. For a topological space $X$ equipped with a closed partial order, the stochastic order on $\mathcal{P}(X)$ is defined by $\mu \leq \nu$ if $\mu(U) \leq \nu(U)$ for each open upper set $U$.

Proposition 3.4. Let $X$ be a metric space equipped with a closed partial order. Then the following are equivalent for $\mu, \nu \in \mathcal{P}(X)$ :

(1) $\mu \leq \nu$;

(2) $\mu(A) \leq \nu(A)$ for each closed upper set $A$;

(3) $\mu(B) \leq \nu(B)$ for each upper Borel set $B$.

Proof. Clearly (3) implies both (1) and (2).

$(1) \Rightarrow(3)$ : Let $B=\uparrow B$ be a Borel set. The $A=X \backslash B$ is also a Borel set. Let $\varepsilon>0$. By inner regularity there exists a compact set $K \subseteq A$ such that $\nu(K)>\nu(A)-\varepsilon$. 
By Lemma $3.2 \downarrow K$ is closed, and $K \subseteq \downarrow K \subseteq \downarrow A=A$. Thus $\nu(\downarrow K)>\nu(A)-\varepsilon$. The complement $U$ of $\downarrow K$ is an open upper set. Taking complements we obtain

$$
\begin{aligned}
\mu(B) & =1-\mu(A) \leq 1-\mu(\downarrow K)=\mu(U) \leq \nu(U)=1-\nu(\downarrow K) \\
& <1-\nu(A)+\varepsilon=\nu(B)+\varepsilon .
\end{aligned}
$$

Since $\mu(B)<\nu(B)+\varepsilon$ for all $\varepsilon>0$, we conclude $\mu(B) \leq \nu(B)$.

$(2) \Rightarrow(3)$ : We can approximate any Borel upper set $B$ arbitrarily closely from the inside with compact subsets $K$ and their upper sets $\uparrow K$ will be closed sets that are at least as good approximations. The Borel measure $\nu$ dominates $\mu$ on these closed upper sets and hence also in the limiting case of $B$.

Remark 3.5. By taking complements one determines that each of the preceding equivalences has an equivalent version for lower sets with the inequalities in (2) and (3) reversed.

We turn now to functional characterizations of the stochastic order on $\mathcal{P}(X)$ for $X$ a metric space equipped with a closed partial order. In the next proposition, we write $\int_{X} f(x) d \mu(x)$ or simply $\int_{X} f d \mu$ for any Borel function $f: X \rightarrow \mathbb{R}^{+}$and $\mu \in \mathcal{P}(X)$, where the integral is possibly infinite. We say that $f$ is monotone if $x \leq y$ in $X$ implies $f(x) \leq f(y)$.

Proposition 3.6. Let $X$ be a metric space equipped with a closed partial order. Then the following are equivalent for $\mu, \nu \in \mathcal{P}(X)$ :

(1) $\mu \leq \nu$;

(2) for every monotone (bounded) Borel function $f: X \rightarrow \mathbb{R}^{+}, \int_{X} f d \mu \leq \int_{X} f d \nu$;

(3) for every monotone (bounded) lower semicontinuous $f: X \rightarrow \mathbb{R}^{+}, \int_{X} f d \mu \leq$ $\int_{X} f d \nu$.

Proof. The implications that the general case implies the bounded case in items (2) and (3) are trivial.

$(1) \Rightarrow(2)$ : Assume $\mu \leq \nu$ and let $f$ be a non-negative monotone Borel measurable function on $X$. For each $n$, define $\delta_{n}: \mathbb{R}^{+} \rightarrow \mathbb{R}^{+}$by $\delta_{n}(0)=0, \delta_{n}(t)=(i-1) / 2^{n}$ if $(i-1) / 2^{n}<t \leq i / 2^{n}$ for some integer $i, 1 \leq i \leq n 2^{n}$, and $\delta_{n}(t)=n$ for $n<t$. Note that the ascending step function $\delta_{n}$ has finite image contained in $\mathbb{R}^{+}$and that the 
sequence $\delta_{n}$ monotonically increases to the identity map on $\mathbb{R}^{+}$. Hence $f_{n}:=\delta_{n} f$, the composition of $\delta_{n}$ and $f$, monotonically increases to $f$. One verifies directly that the step function $f_{n}$ has an alternative description given by

$$
f_{n}=\sum_{i=1}^{n 2^{n}} \frac{1}{2^{n}} \chi_{\left.\left.f^{-1}(] i / 2^{n}, \infty\right)\right)}
$$

where $\chi_{A}$ is the characteristic function of $A$. Since the sequence $\left\{f_{n}\right\}$ converges pointwise and monotonically to $f$, we conclude that $\int_{X} f d \mu=\lim _{n} \int_{X} f_{n} d \mu$, and similarly for $\nu$. Since $\left.\left.f^{-1}(] i / 2^{n}, \infty\right)\right)$ is an upper Borel set, by Proposition 3.4 $\left.\left.\left.\left.\mu\left(f^{-1}(] i / 2^{n}, \infty\right)\right)\right) \leq \nu\left(f^{-1}(] i / 2^{n}, \infty\right)\right)\right)$ for each $i$, so $\int_{X} f_{n} d \mu \leq \int_{X} f_{n} d \nu$ for each $n$, and thus in the limit $\int_{X} f d \mu \leq \int_{X} f d \nu$.

$(2) \Rightarrow(3)$ : Since a lower semicontinuous function is a Borel measurable function, (3) follows immediately from (2).

$(3) \Rightarrow(1)$ : The characteristic function $\chi_{U}$ is bounded, lower semicontinuous, and monotone for $U$ an open upper set and hence $\mu(U)=\int_{X} \chi_{U} d \mu \leq \int_{X} \chi_{U} d \nu=\nu(U)$.

Call a real function $f$ on a partially ordered set $X$ antitone if it is order reversing, i.e., $x \leq y$ implies $f(x) \geq f(y)$.

Corollary 3.7. Let $X$ be a metric space equipped with a closed partial order. Then the following are equivalent for $\mu, \nu \in \mathcal{P}(X)$ :

(1) $\nu \leq \mu$;

(2) for every antitone (bounded) Borel function $f: X \rightarrow \mathbb{R}^{+}, \int_{X} f d \mu \leq \int_{X} f d \nu$;

(3) for every antitone (bounded) lower semicontinuous $f: X \rightarrow \mathbb{R}^{+}, \int_{X} f d \mu \leq$ $\int_{X} f d \nu$.

Proof. Every partially ordered set has a dual order, namely the converse $\geq$ of $\leq$ is taken for the partial order. Let $X^{\text {od }}$ denote the order dual of $X$. Note that a subset $A$ of $X$ is an upper set in $(X, \leq)$ if and only if it is a lower set in $X^{\text {od }}$. Using Remark 3.5, one sees that $\mu \leq \nu$ with respect to $(X, \leq)$ if and only if $\nu \leq \mu$ with respect to $X^{\text {od }}$. Since antitone functions convert to monotone functions in the order dual of $X$, the corollary follows from applying the previous proposition to the order dual. 
Finally we consider sufficient conditions for one to define the stochastic order in terms of continuous monotone functions.

Proposition 3.8. Suppose that $(X, d)$ is a metric space equipped with a closed partial order satisfying the property that given $x \leq y$ and $x_{1} \in X$, there exists $y_{1} \geq x_{1}$ such that $d\left(y, y_{1}\right) \leq d\left(x, x_{1}\right)$. Then for $\mu, \nu \in \mathcal{P}(X)$ the following are equivalent:

(1) $\mu \leq \nu$;

(2) For every continuous (bounded) monotone $f: X \rightarrow \mathbb{R}^{+}, \int_{X} f d \mu \leq \int_{X} f d \nu$;

(3) For every continuous (bounded) antitone $f: X \rightarrow \mathbb{R}^{+}, \int_{X} f d \nu \leq \int_{X} f d \mu$.

Proof. That (1) implies (2) follows from Proposition 3.6 and (1) implies (3) by Corollary 3.7.

$(3) \Rightarrow(1)$ : Let $V$ be an open lower set with complement $A$, a closed upper set. For each $n \in \mathbb{N}$, define $f_{n}: X \rightarrow[0,1]$ by $f_{n}(x)=\min \{n d(x, A), 1\}$ and note that $f_{n}$ is a continuous function into $[0,1]$. To show $f_{n}$ is antitone, we note for any $x \leq y$ and $x_{1} \in A$, there exists $y_{1} \geq x_{1}$ such that $d\left(y, y_{1}\right) \leq d\left(x, x_{1}\right)$. It follows from $x_{1} \leq y_{1}$ that $y_{1} \in A$, hence $d(y, A) \leq d\left(x, x_{1}\right)$, and thus $d(y, A) \leq d(x, A)$ since $x_{1}$ was an arbitrary point of $A$. Hence

$$
f_{n}(y)=\min \{n d(y, A), 1\} \leq \min \{n d(x, A), 1\}=f_{n}(x) .
$$

It follows directly from the definition of $f_{n}$ that the sequence $\left\{f_{n}\right\}$ is an monotonically increasing sequence with supremum $\chi_{V}$. Thus

$$
\left.\nu(V)=\int_{X} \chi_{V} d \nu=\lim _{n} \int_{X} f_{n} d \nu \leq \lim _{n} \int_{X} f_{n} d \mu=\int_{X} \chi_{V} d \mu\right)=\mu(V) .
$$

Since $V$ was an arbitrary open lower set, $\mu \leq \nu$ by Remark 3.5 .

$(2) \Rightarrow(1)$ : Property (2) implies that $\int_{X} f d \mu \leq \int_{X} f d \nu$ for every continuous antitone function $f: X^{o d} \rightarrow \mathbb{R}^{+}$. By the preceding paragraph $\nu \leq \mu$ with respect to $X^{\text {od }}$, i.e., $\mu \leq \nu$ with respect to $(X, \leq)$.

Definition 3.9. A topological space equipped with a closed order is called monotone normal if given a closed upper set $A$ and a closed lower set $B$ such that $A \cap B=\emptyset$, there exist an open upper set $U \supseteq A$ and an open lower set $V \supseteq B$ such that $U \cap V=\emptyset$. 
Remark 3.10. Assume that $(X, d)$ satisfies the property stated in Proposition 3.8 and also its dual version that given $x \leq y$ and $y_{1} \in X$, there is an $x_{1} \in X$ such that $x_{1} \leq y_{1}$ and $d\left(x, x_{1}\right) \leq d\left(y, y_{1}\right)$. Then $(X, \leq)$ is monotone normal as in the above definition. Indeed, for any closed upper set $A$ and any closed lower set $B$ with $A \cap B=\emptyset$, one can easily verify that the open sets

$$
U:=\{x \in X: d(x, A)<d(x, B)\} \quad \text { and } \quad V:=\{x \in X: d(x, A)>d(x, B)\}
$$

satisfy $U \supseteq A, V \supseteq B$ and $U \cap V=\emptyset$. One deduces that $U$ is an upper set and $V$ a lower from the hypothesized property and its dual. Also, we remark that an open cone in a Banach space as considered in Section 5 satisfies the above two properties (see Remark 5.4).

Proposition 3.11. Suppose that $(X, d)$ is a metric space equipped with a closed partial order for which the space is monotone normal. Then for $\mu, \nu \in \mathcal{P}(X)$ the following are equivalent:

(1) $\mu \leq \nu$;

(2) For every continuous (bounded) monotone $f: X \rightarrow \mathbb{R}^{+}, \int_{X} f d \mu \leq \int_{X} f d \nu$.

Proof. In light of Proposition 3.6 we need only show condition (2) implies condition (1). Suppose there exists some open upper set $U$ such that $\nu(U)<\mu(U)$. By inner regularity there exists a compact set $K \subseteq U$ such that $\nu(U)<\mu(K) \leq \mu(U)$. The closed upper set $A=\uparrow K \subseteq U$ also satisfies $\nu(U)<\mu(A) \leq \mu(U)$. Since $X$ is monotone normal, a modification of the usual proof of Urysohn's Lemma yields a continuous monotone function $f: X \rightarrow[0,1]$ such that $f(A)=1$ and $f(X \backslash U)=0$; see for example [5, Exercise VI-1.16]. We then have

$$
\mu(A)=\int_{X} \chi_{A} d \mu \leq \int_{X} f d \mu \leq \int_{X} f d \nu \leq \int_{X} \chi_{U} d \nu=\nu(U),
$$

a contradiction to our choice of $A$.

\section{NORMAL CONES}

Let $E$ be a Banach space containing an open cone $C$ such that its closure $\bar{C}$ is a proper cone, i.e., $\bar{C} \cap(-\bar{C})=\{0\}$. The cone $\bar{C}$ defines a closed partial order on $E$ by $x \leq y$ if $y-x \in \bar{C}$. The cone $\bar{C}$ is called normal if there is a constant $K$ such that $0 \leq x \leq y$ implies $\|x\| \leq K\|y\|$. 
For $x \leq y$ in $E$, the order interval $[x, y]$ is given by

$$
[x, y]:=\{w \in E: x \leq w \leq y\}=(x+\bar{C}) \cap(y-\bar{C})
$$

Note that $(x+C) \cap(y-C)$ is an open subset contained in $[x, y]$. A subset $B$ is order convex if $[x, y] \subseteq B$, wherever $x, y \in B$ and $x \leq y$. An alternative formulation of normality postulates the existence of a basis of order convex neighborhoods at 0 and hence by translation at all points (see Section 19.1 of [2]); here neighborhood of $x$ means a subset containing $x$ in its interior.

Proposition 4.1. Let $E$ be a separable Banach space with an open cone $C$ such that $\bar{C}$ is normal. Then restricted to $C$, the $\sigma$-algebra generated by all its open upper sets is the Borel algebra of $C$.

Proof. Let $\mathcal{A}$ denote the $\sigma$-algebra of subsets of $C$ generated by the collection of all open upper sets contained in $C$. Fix some point $u \in C$. Let $x \in C$. Set $r_{n}=1 / n$ for $n \in \mathbb{N}$. Then for each $n, x \in\left(x-r_{n} u\right)+C$, an open upper set, and $\uparrow x=$ $\bigcap_{n}\left[\left(x-r_{n} u\right)+C\right]$. In fact, for any $y$ in the intersection $y-\left(x-r_{n} u\right)=(y-x)-r_{n} u \in C$ and hence the limit $y-x$ is in $\bar{C}$, i.e., $y \in x+\bar{C}=\uparrow x$. The converse inclusion is obvious since $r_{n} u+\bar{C} \subseteq C$ so that $x+\bar{C} \subseteq\left(x-r_{n} u\right)+C$. Thus $\uparrow x$ is a countable intersection of open upper sets, hence in $\mathcal{A}$.

Since $\downarrow x$ is closed in $E, C \cap(E \backslash \downarrow x)$ is an open upper set. Thus its complement in $C$, which is $C \cap \downarrow x$ is in $\mathcal{A}$. Hence for $x \leq y$ in $C$, we note that $[x, y]=\uparrow x \cap \downarrow y=$ $\uparrow x \cap C \cap \downarrow y \in \mathcal{A}$.

Now let $U$ be a nonempty open subset of $C$. Using the alternative characterization of normality, we may pick for each $x \in U$ an order convex neighborhood $N_{x}$ of $x$ that is contained in $U$. For some $\varepsilon$ small enough $x-\varepsilon u, x+\varepsilon u \in N_{x}$, and hence the order interval $[x-\varepsilon u, x+\varepsilon u] \subseteq N_{x}$. Let $B_{x}:=(x-\varepsilon u+C) \cap(x+\varepsilon u-C)$, an open subset contained in $[x-\varepsilon u, x+\varepsilon u]$. The collection $\left\{B_{x}: x \in C\right\}$ is an open cover of $U$, which by the separability of $E$ (and hence $U$ ) has a countable subcover $\left\{B_{x_{n}}\right\}$. The corresponding $\left[x_{n}-\varepsilon_{n} u, x_{n}+\varepsilon_{n} u\right]$ then also form a countable cover of $U$, and since from the preceding paragraph each order interval is in $\mathcal{A}$, it follows that $U \in \mathcal{A}$. Thus $\mathcal{A}$ contains all open sets of $C$, and hence must be the Borel algebra. 
We next recall E. Dynkin's $\pi-\lambda$ theorem. Let $X$ be a set. A $\pi$-system is a collection of subsets of $X$ closed under finite intersection. A $\lambda$-system is a collection with $X$ as a member that is closed under complementation and under countable unions of pairwise disjoint members of the system. An important observation is that a $\lambda$-system that is also a $\pi$-system is a $\sigma$-algebra.

Theorem 4.2. (Dynkin's $\pi-\lambda$ Theorem) If a $\pi$-system is contained in a $\lambda$-system, then the $\sigma$-algebra generated by the $\pi$-system is contained in the $\lambda$-system.

The stochastic order on $\mathcal{P}(X)$ for a metric space $X$ equipped with a closed order is easily seen to be reflexive and transitive, but anti-symmetry is much more difficult to derive. We now have available the tools we need to show for the open cone $C$ that the stochastic order on $\mathcal{P}(C)$ is a partial order.

Theorem 4.3. Let $E$ be a Banach space containing an open cone $C$ such that $\bar{C}$ is a normal cone. Then the stochastic order on $\mathcal{P}(C)$ is a partial order.

Proof. We first consider the case that $E$ is separable. Let $\mu, \nu \in \mathcal{P}(C)$ be such that $\mu \leq \nu$ and $\nu \leq \mu$. We consider the set $\mathcal{A}$ of all Borel sets $B$ such that $\mu(B)=\nu(B)$. By definition of the stochastic order, $U \in \mathcal{A}$ for each open upper set $U$, and the collection of open upper sets is closed under finite intersection, i.e., is a $\pi$-system. Since $\mu$ and $\nu$ are $\sigma$-additive measures, it follows that the collection $\mathcal{A}$ is closed under complementation and union of pairwise disjoint countable families, so $\mathcal{A}$ is a $\lambda$-system. By Dynkin's $\pi-\lambda$ theorem the $\sigma$-algebra generated by the open upper sets is contained in $\mathcal{A}$, but by Proposition 4.1 this is the Borel algebra. Hence $\mu=\nu$ on the Borel algebra, that is to say $\mu=\nu$.

We turn now to the general case in which $E$ may not be separable. In this case, however, both $S_{\mu}$, the support of $\mu$, and $S_{\nu}$, the support of $\nu$, are separable (Proposition 2.1). Then also the smallest closed Banach subspace $F$ containing $S_{\mu} \cup S_{\nu}$ will be separable, and the restrictions $\left.\mu\right|_{F},\left.\nu\right|_{F} \in \mathcal{P}(C \cap F)$. Since $C \cap F$ is an open cone in $F$ with closure a normal cone, by the first part of the proof $\mu(B)=\nu(B)$ for all Borel subsets contained in $C \cap F$. Since $S_{\mu} \cup S_{\nu} \subseteq C \cap F$, for any Borel set $B \subseteq C$,

$$
\mu(B)=\mu\left(B \cap S_{\mu}\right)=\mu\left(B \cap\left(S_{\mu} \cup S_{\nu}\right)\right)=\nu\left(B \cap\left(S_{\mu} \cup S_{\nu}\right)\right)=\nu\left(B \cap S_{\nu}\right)=\nu(B) .
$$

Thus $\mu=\nu$. 
Remark 4.4. The techniques of the proof readily extend to any open upper set of $E$, in particular to $E$ itself. Indeed, Proposition 4.1 and Theorem 4.3 hold when restricted to any open upper set in place of $C$. So the stochastic order on $\mathcal{P}(E)$ arising from the conic order of $E$ is also a partial order.

\section{The Thompson Metric}

We continue in the setting that $E$ is a Banach space and $C$ is an open cone with its closure $\bar{C}$ a normal cone. A. C. Thompson [14] has proved that $C$ is a complete metric space with respect to the Thompson part metric defined by

$$
d_{T}(x, y)=\max \{\log M(x / y), \log M(y / x)\}
$$

where $M(x / y):=\inf \{\lambda>0: x \leq \lambda y\}=|x|_{y}$. Furthermore, the metric topology on $C$ arising from the Thompson metric agrees with relative topology inherited from $E$.

The contractivity of addition in $C$ with respect to the Thompson metric has been observed in various settings and studied in some detail in [8]. We need only the basic formulation.

Lemma 5.1. Addition is contractive on $C$ with respect to the Thompson metric in the sense that for all $x, y, z \in C, d_{T}(x+z, y+z) \leq d_{T}(x, y)$.

Remark 5.2. The fact that the Thompson metric is complete allows us to deduce from Proposition 2.1 for $E$ separable that $\mathcal{P}(C)$ consists of all Borel probability measures and for $E$ an arbitrary Banach space that $\mathcal{P}(C)$ consists of the $\tau$-additive probability measures.

Proposition 5.3. The cone $C$ equipped with the Thompson metric satisfies the property that given $x \leq y$ and $x_{1} \in C$, there exists $y_{1} \geq x_{1}$ such that $d_{T}\left(y, y_{1}\right) \leq d_{T}\left(x, x_{1}\right)$. Hence for $\mu, \nu \in \mathcal{P}(C), \mu \leq \nu$ in the stochastic order if and only if for every continuous (bounded) monotone $f: X \rightarrow \mathbb{R}^{+}, \int_{X} f d \mu \leq \int_{X} f d \nu$.

Proof. Suppose $x \leq y$ and $x_{1} \in C$. The contractivity of the Thompson metric (Lemma 5.1) implies for $y_{1}=x_{1}+(y-x)$ that

$$
d_{T}\left(y, y_{1}\right)=d_{T}\left(x+(y-x), x_{1}+(y-x)\right) \leq d_{T}\left(x, x_{1}\right) .
$$

The last assertion of the proposition now follows from Proposition 3.8. 
Remark 5.4. Here is a second proof of Proposition 5.3. Assume that $x \leq y$ in $C$. For every $x_{1} \in C$ let $\alpha:=d_{T}\left(x, x_{1}\right)$ so that $e^{-\alpha} x \leq x_{1} \leq e^{\alpha} x$. Set $y_{1}:=e^{\alpha} y$; then $y_{1} \geq e^{\alpha} x \geq x_{1}$ and $y \leq y_{1}=e^{\alpha} y$, so $d_{T}\left(y, y_{1}\right) \leq \alpha=d_{T}\left(x, x_{1}\right)$. Similarly one can show the dual version mentioned in Remark 3.10. For every $y_{1} \in C$ let $\beta:=d_{T}\left(y, y_{1}\right)$ and $x_{1}:=e^{-\beta} x$; then $x_{1} \leq e^{-\beta} y \leq y_{1}$ and $e^{-\beta} x=x_{1} \leq x$ so that $d_{T}\left(x, x_{1}\right) \leq \beta=d_{T}\left(y, y_{1}\right)$.

Recall that one of the characterizations of the weak topology on any metric space, in particular on $\mathcal{P}(C)$, is that a net $\mu_{\alpha} \rightarrow \mu$ weakly if and only if $\lim _{\alpha} \int_{C} f d \mu_{\alpha} \rightarrow \int_{C} f d \mu$ for all continuous bounded functions into $\mathbb{R}$ (or $\mathbb{R}^{+}$); see [1].

Proposition 5.5. The stochastic partial order is a closed subset of $\mathcal{P}(C) \times \mathcal{P}(C)$ endowed with the product weak topology.

Proof. Let $\mu_{\alpha} \rightarrow \mu$ and $\nu_{\alpha} \rightarrow \nu$ weakly in $\mathcal{P}(C)$, where $\mu_{\alpha} \leq \nu_{\alpha}$ for each $\alpha$. From Proposition 5.3 for $f: C \rightarrow \mathbb{R}^{+}$continuous bounded and monotone

$$
\int_{C} f d \mu=\lim _{\alpha} \int_{C} f d \mu_{\alpha} \leq \lim _{\alpha} \int_{C} f d \nu_{\alpha}=\int_{C} f d \nu
$$

Thus again from Proposition $5.3, \mu \leq \nu$.

Let $(X, \mathcal{M})$ be a measure space, a set $X$ equipped with a $\sigma$-algebra $\mathcal{M}$, and $(Y, d)$ a metric space. A function $f: X \rightarrow Y$ is measurable if $f^{-1}(A) \in \mathcal{M}$ whenever $A \in$ $\mathcal{B}(Y)$. For $f$ to be measurable, it suffices that $f^{-1}(U) \in \mathcal{M}$ for each open subset $U$ of $Y$. Hence continuous functions are measurable in the case $X$ is a metrizable space and $\mathcal{M}=\mathcal{B}(X)$, the Borel algebra. A measurable map $f: X \rightarrow Y$ between metric spaces induces the push-forward map $f_{*}: \mathcal{P}(X) \rightarrow \mathcal{P}(Y)$ defined by $f_{*}(\mu)(B)=\mu\left(f^{-1}(B)\right)$ for $\mu \in \mathcal{P}(X)$ and $B \in \mathcal{B}(Y)$. Note for $f$ continuous that $\operatorname{supp}\left(f_{*}(\mu)\right)=f(\operatorname{supp}(\mu))^{-}$, the closure of the image of the support of $\mu$.

Let $(X, d)$ be a complete metric space, and for $p \in[1, \infty)$ let $\mathcal{P}^{p}(X):=\{\mu \in \mathcal{P}(X)$ : $\left.\int_{X} d(x, y)^{p} d \mu(y)<\infty\right\}$, the set of $\tau$-additive Borel probability measures on $X$ with finite $p$ th moment (defined independently of the choice of $x \in X$ ). The $p$-Wasserstein metric $d_{p}^{W}$ on $\mathcal{P}^{p}(X)$ is defined by

$$
d_{p}^{W}(\mu, \nu):=\left[\inf _{\pi \in \Pi(\mu, \nu)} \int_{X \times X} d(x, y)^{p} d \pi(x, y)\right]^{1 / p}, \quad \mu, \nu \in \mathcal{P}^{p}(X),
$$


where $\Pi(\mu, \nu)$ is the set of all couplings for $\mu, \nu$, i.e., $\pi \in \mathcal{P}(X \times X)$ whose marginals are $\mu$ and $\nu$.

Recall (see, e.g., [13) that $\mathcal{P}^{p}(X)$ is a complete metric space with the metric $d_{p}^{W}$, and that the Wasserstein convergence implies weak convergence. Hence we have the following corollary of the preceding proposition.

Corollary 5.6. The stochastic partial order is a closed subset of $\mathcal{P}^{1}(C) \times \mathcal{P}^{1}(C)$ endowed with the product Wasserstein topology (induced by $d_{1}^{W}$ ).

We recall the notion of a contractive barycentric map.

Definition 5.7. Let $(X, d)$ be a complete metric space. A map $\beta: \mathcal{P}^{1}(X) \rightarrow X$ is called a contractive barycentric map if

(i) $\beta\left(\delta_{x}\right)=x$ for all $x \in X$;

(ii) $d(\beta(\mu), \beta(\nu)) \leq d_{1}^{W}(\mu, \nu)$ for all $\mu, \nu \in \mathcal{P}^{1}(X)$.

For a closed partial order $\leq$ on $X, \beta: \mathcal{P}^{1}(X) \rightarrow X$ is said to be monotonic if $\beta(\mu) \leq \beta(\nu)$, whenever $\mu \leq \nu$.

A complete partially ordered metric space equipped with a monotonic contractive barycenter has become an important object of study in recent years.

We consider the semigroup of mappings on $\psi: C \rightarrow C$ satisfying $x \leq \psi(x)$ for all $x \in C$. For instance, every translation $\tau_{a}(x)=a+x, a \in \bar{C}$, satisfies this condition and also is non-expansive for the Thompson metric.

Corollary 5.8. Let $\psi: C \rightarrow C$ be a Lipschitzian map with respect to the Thompson metric $d_{T}$ such that $x \leq \psi(x)$ for all $x \in C$. Then for every $\mu \in \mathcal{P}^{1}(C)$, we have $\psi_{*} \mu \in \mathcal{P}^{1}(C)$ and $\mu \leq \psi_{*} \mu$. If further, $\beta: \mathcal{P}^{1}(C) \rightarrow C$ is a monotonic barycentric map, then $\beta(\mu) \leq \beta\left(\psi_{*} \mu\right)$ for any $\mu \in \mathcal{P}^{1}(C)$.

Proof. Let $\mu=\frac{1}{n} \sum_{j=1}^{n} \delta_{x_{j}}$ be a finitely supported uniform measure on $C$. From $x_{j} \leq \psi\left(x_{j}\right)$ for all $j$, we have

$$
\mu=\frac{1}{n} \sum_{j=1}^{n} \delta_{x_{j}} \leq \frac{1}{n} \sum_{j=1}^{n} \delta_{\psi\left(x_{j}\right)}=\psi_{*} \mu .
$$

Now for $\mu \in \mathcal{P}^{1}(\mathbb{P})$, pick a sequence $\mu_{n}$ of finitely supported uniform measures converging to $\mu$ from below for the Wasserstein metric associated to the Thompson 
metric ([7, Theorem 4.7]). Then

$$
\mu \leq \mu_{n} \leq \psi_{*} \mu_{n} \rightarrow \psi_{*} \mu
$$

as $n \rightarrow \infty$ and hence $\mu \leq \psi_{*} \mu$, by the previous corollary.

\section{ORDER-COMPLETENESS}

In this section we always assume that the Banach space $E$ is finite-dimensional (hence separable) and, as in Section $4, C$ is an open cone in $E$ whose closure $\bar{C}$ is a proper cone. Note (see Section 19.1 of [2]) that the finite dimensionality assumption automatically implies that $\bar{C}$ is a normal cone. We consider $C$ as a complete metric space equipped with the Thompson part metric $d_{T}$ and the $p$-Wasserstein metric $d_{p}^{W}$ on $\mathcal{P}^{p}(C), 1 \leq p<\infty$, given in (5.1) with $d=d_{T}$.

The next elementary lemma is given just for completeness.

Lemma 6.1. (1) For each $x, y \in C$, the order interval $[x, y]=(x+\bar{C}) \cap(y-\bar{C})$ is a compact subset of $C$.

(2) For any $u \in C, \bigcup_{k=1}^{\infty}\left[k^{-1} u, k u\right]=C$.

Proof. (1): Since $x+\bar{C} \subset C+\bar{C} \subset C,[x, y] \subset C$. It is also clear that $[x, y]$ is a closed subset of $E$. Since $\bar{C}$ is a normal cone, we see that if $z \in[x, y]$ then $\|z\| \leq K\|y\|$. Hence, $[x, y]$ is a bounded closed subset of $E$. Since $E$ is finite-dimensional, $[x, y]$ is compact in $E$ and so is in $(C, d)$.

(2): Let $u, x \in C$. For $k \in \mathbb{N}$ sufficiently large, $x-k^{-1} u \in C$ and $u-k^{-1} x \in C$ so that $x \in\left(k^{-1} u+C\right) \cap(k u-C)$. Therefore, $x \in\left[k^{-1} u, k u\right]$, which implies the assertion.

Before showing order-completeness, it is convenient to derive the compactness of order intervals in $\mathcal{P}(C)$ as well as in $\mathcal{P}^{p}(C)$.

Proposition 6.2. Let $\nu_{1}, \nu_{2} \in \mathcal{P}(C)$ with $\nu_{1} \leq \nu_{2}$.

(1) The order interval $\left[\nu_{1}, \nu_{2}\right]:=\left\{\mu \in \mathcal{P}(C): \nu_{1} \leq \mu \leq \nu_{2}\right\}$ is compact in the weak topology.

(2) Let $1 \leq p<\infty$. If $\nu_{1}, \nu_{2} \in \mathcal{P}^{p}(C)$, then $\left[\nu_{1}, \nu_{2}\right] \subset \mathcal{P}^{p}(C)$ and it is compact in the $d_{p}^{W}$-topology. 
Proof. (1): Choose any $u \in C$. For every $\epsilon>0$ Lemma 6.1(2) implies that there exists $k \in \mathbb{N}$ such that $\left(\nu_{1}+\nu_{2}\right)\left(C \backslash\left[k^{-1} u, k u\right]\right)<\epsilon$. We write $C \backslash\left[k^{-1} u, k u\right]=U_{k} \cup V_{k}$, where $U_{k}:=\{x \in C: x \not \leq k u\}$ and $V_{k}:=\left\{x \in C: x \nsupseteq k^{-1} u\right\}$. It is clear that $U_{k}$ is an upper open set while $V_{k}$ is a lower open set. Hence, if $\mu \in\left[\nu_{1}, \nu_{2}\right]$, then we have

$$
\begin{aligned}
\mu\left(C \backslash\left[k^{-1} u, k u\right]\right) & \leq \mu\left(U_{k}\right)+\mu\left(V_{k}\right) \leq \nu_{2}\left(U_{k}\right)+\nu_{1}\left(V_{k}\right) \\
& \leq\left(\nu_{1}+\nu_{2}\right)\left(C \backslash\left[k^{-1} u, k u\right]\right)<\epsilon
\end{aligned}
$$

(for $\mu\left(V_{k}\right) \leq \nu_{1}\left(V_{k}\right)$, see Remark 3.5). By Lemma 6.1 (1), this says that $\left[\nu_{1}, \nu_{2}\right]$ is tight, and so it is relatively compact in $\mathcal{P}(C)$ in the weak topology due to Prohorov's theorem (see [1]). Since $\left[\nu_{1}, \nu_{2}\right]$ is closed in the weak topology by Proposition [5.5, $\left[\nu_{1}, \nu_{2}\right]$ is compact in the weak topology.

(2): Next, assume that $\nu_{1}, \nu_{2} \in \mathcal{P}^{p}(C)$ for $p \in[1, \infty)$. First we prove the following "tightness" condition:

$$
\lim _{R \rightarrow \infty} \sup _{\mu \in\left[\nu_{1}, \mu_{2}\right]} \int_{d(x, u)>R} d(x, u)^{p} d \mu(x)=0
$$

for some $u \in C$. Choose any $u \in C$. For every $R \geq 0$ set

$$
\begin{aligned}
& U_{R}:=\left\{x \in C: M(x / u)>e^{R}, M(x / u) \geq M(u / x)\right\}, \\
& V_{R}:=\left\{x \in C: M(u / x)>e^{R}, M(x / u)<M(u / x)\right\} .
\end{aligned}
$$

Then it is immediate to see that

$$
\{x \in C: d(x, u)>R\}=U_{R} \cup V_{R} \text { (disjoint sum). }
$$

Hence, for any $\mu \in \mathcal{P}(C)$ we have

$$
\int_{d(x, u)>R} d(x, u)^{p} d \mu(x)=\int_{C} 1_{U_{R}}(x) d(x, u)^{p} d \mu(x)+\int_{C} 1_{V_{R}}(x) d(x, u)^{p} d \mu(x) .
$$

When $x \in U_{R}$ and $x \leq y \in C$, since $M(x / u) \leq M(y / u)$ and $M(u / x) \geq M(u / y)$, we find that $M(y / u) \geq M(x / u)>e^{R}$ and $M(y / u) \geq M(u / y)$ so that $y \in U_{R}$. Therefore, $U_{R}$ is an upper Borel set. Moreover,

$$
d(x, u)=\log M(x / u) \leq \log M(y / u)=d(y, u) .
$$

Hence it follows that $x \in C \mapsto 1_{U_{R}}(x) d(x, u)^{p}$ is a monotone Borel function. When $x \in V_{R}$ and $x \geq y \in C$, since $M(x / u) \geq M(y / u)$ and $M(u / x) \leq M(u / y), M(u / y) \geq$ 
$M(u / x)>e^{R}$ and $M(y / u)<M(u / y)$ so that $y \in V_{R}$. Therefore, $V_{R}$ is a lower open set and

$$
d(x, u)=\log M(u / x) \leq \log M(u / y)=d(y, u) .
$$

Hence we see that $x \in C \mapsto 1_{V_{R}}(x) d(x, u)^{p}$ is an antitone Borel function. If $\mu \in$ $\left[\nu_{1}, \nu_{2}\right]$, then by Proposition 3.6 and Corollary 3.7 applied to the right-hand side of (6.3) we obtain

$$
\begin{aligned}
\int_{d(x, u)>R} d(x, u)^{p} d \mu(x) & \leq \int_{C} 1_{U_{R}}(x) d(x, u)^{p} d \nu_{2}(x)+\int_{C} 1_{V_{R}}(x) d(x, u)^{p} d \nu_{1}(x) \\
& \leq \int_{d(x, u)>R} d(x, u)^{p} d\left(\nu_{1}+\nu_{2}\right)(x) \longrightarrow 0
\end{aligned}
$$

as $R \rightarrow \infty$, since $\int_{C} d(x, u)^{p} d\left(\nu_{1}+\nu_{2}\right)(x)<\infty$. Hence (6.2) has been proved, which in particular implies that $\left[\nu_{1}, \nu_{2}\right] \subseteq \mathcal{P}^{p}(C)$. Moreover, from a basic fact on the convergence in Wasserstein spaces [15, Theorem 7.12], we see that $\left[\nu_{1}, \nu_{2}\right]$ is compact in the $d_{p}^{W}$-topology. Indeed, for every sequence $\left\{\mu_{n}\right\}$ in $\left[\nu_{1}, \nu_{2}\right]$, from the assertion (1) one can choose a subsequence $\left\{\mu_{n(m)}\right\}$ such that $\mu_{n(m)} \rightarrow \mu$ weakly for some $\mu \in \mathcal{P}(C)$. Hence, it follows from [15, Theorem 7.12] that $\mu \in \mathcal{P}^{p}(C)$ and $d_{p}^{W}\left(\mu_{n(m)}, \mu\right) \rightarrow 0$. Note that the limit $\mu$ is in $\left[\nu_{1}, \nu_{2}\right]$, since the $d_{p}^{W}$-convergence implies the weak convergence. Thus, $\left[\nu_{1}, \nu_{2}\right]$ is $d_{p}^{W}$-compact.

The next proposition gives the order-completeness (or a monotone convergence property) of the stochastic order on $\mathcal{P}(C)$ in the weak topology.

Proposition 6.3. Let $\mu_{n}, \nu \in \mathcal{P}(C)$ for $n \in \mathbb{N}$.

(1) If $\mu_{1} \leq \mu_{2} \leq \cdots \leq \nu$, then there exists a $\mu \in \mathcal{P}(C)$ such that $\mu_{n} \leq \mu \leq \nu$ for all $n$ and $\mu_{n} \rightarrow \mu$ weakly.

(2) If $\mu_{1} \geq \mu_{2} \geq \cdots \geq \nu$, then there exists a $\mu \in \mathcal{P}(C)$ such that $\mu_{n} \geq \mu \geq \nu$ for all $n$ and $\mu_{n} \rightarrow \mu$ weakly.

Proof. (1): Since $\left\{\mu_{n}\right\} \subset\left[\mu_{1}, \nu\right]$ and Proposition 6.2(1) says that $\left[\mu_{1}, \nu\right]$ is compact in the weak topology, to see that $\mu_{n} \rightarrow \mu$ weakly for some $\mu \in \mathcal{P}(C)$, it suffices to prove that a weak limit point of $\left\{\mu_{n}\right\}$ is unique. Now, let $\mu, \mu^{\prime} \in \mathcal{P}(C)$ be weak limit points of $\left\{\mu_{n}\right\}$, so there are subsequences $\left\{\mu_{n(l)}\right\}$ and $\left\{\mu_{n(m)}\right\}$ such that $\mu_{n(l)} \rightarrow \mu$ and $\mu_{n(m)} \rightarrow \mu^{\prime}$ weakly. Let $f: C \rightarrow[0, \infty)$ be any continuous bounded and monotone 
function. Since $\int_{C} f d \mu_{n}$ is increasing in $n$ by Proposition 5.3, we have

$$
\int_{C} f d \mu=\lim _{l} \int_{C} f d \mu_{n(l)}=\lim _{m} \int_{C} f d \mu_{n(m)}=\int_{C} f d \mu^{\prime} .
$$

This implies by Proposition 5.3 again that $\mu \leq \mu^{\prime}$ and $\mu^{\prime} \leq \mu$ so that $\mu=\mu^{\prime}$ by Theorem 4.3. Therefore $\mu_{n} \rightarrow \mu \in \mathcal{P}(C)$ weakly. Moreover, since $\int_{C} f d \mu_{n} \leq$ $\int_{C} f d \mu \leq \int_{C} f d \nu$ for every continuous bounded and monotone function $f \geq 0$ on $C$, we have $\mu_{n} \leq \mu \leq \nu$ for all $n$.

(2): The proof is similar to the above with a slight modification.

The next proposition gives the order-completeness of the stochastic order restricted on $\mathcal{P}^{p}(C)$ in the $d_{p}^{W}$-convergence.

Proposition 6.4. Let $1 \leq p<\infty$ and $\mu_{n}, \nu \in \mathcal{P}^{p}(C)$ for $n \in \mathbb{N}$.

(1) If $\mu_{1} \leq \mu_{2} \leq \cdots \leq \nu$, then there exists a $\mu \in \mathcal{P}^{p}(C)$ such that $\mu_{n} \leq \mu \leq \nu$ for all $n$ and $d_{p}^{W}\left(\mu_{n}, \mu\right) \rightarrow 0$.

(2) If $\mu_{1} \geq \mu_{2} \geq \cdots \geq \nu$, then there exists a $\mu \in \mathcal{P}^{p}(C)$ such that $\mu_{n} \geq \mu \geq \nu$ for all $n$ and $d_{p}^{W}\left(\mu_{n}, \mu\right) \rightarrow 0$.

Proof. For both assertions (1) and (2), by Proposition 6.2(2) it suffices to prove that a $d_{p}^{W}$-limit point of $\left\{\mu_{n}\right\}$ is unique. Since the $d_{p}^{W}$-convergence implies the weak convergence, this is immediate from the proof of Proposition 6.3.

Corollary 6.5. Let $\mu, \mu_{n} \in \mathcal{P}(C), n \in \mathbb{N}$. Then $\mu_{n}$ weakly converges to $\mu$ increasingly (resp. decreasingly) in the stochastic order if and only if $\int_{C} f d \mu_{n}$ increases (resp. decreases) to $\int_{C} f d \mu$ for every continuous bounded and monotone $f: C \rightarrow \mathbb{R}^{+}$. Moreover, if $\mu, \mu_{n} \in \mathcal{P}^{p}(C)$ where $1 \leq p<\infty$, then the above conditions are also equivalent to $\mu_{n}$ converges to $\mu$ in the metric $d_{p}^{W}$ increasingly (resp. decreasingly) in the stochastic order.

Proof. Assume that for any $f: C \rightarrow \mathbb{R}^{+}$as stated above, $\int_{C} f d \mu_{n}$ increases (resp. decreases) to $\int_{C} f d \mu$. Then by Proposition [5.3, $\mu_{1} \leq \mu_{2} \leq \cdots \leq \mu\left(\mu_{1} \geq \mu_{2} \geq\right.$ $\cdots \geq \mu)$. By Proposition 6.3 there exists a $\mu_{0} \in \mathcal{P}(C)$ such that $\mu_{n} \rightarrow \mu_{0}$ weakly. By assumption, $\int_{C} f d \mu=\int_{C} f d \mu_{0}$ for any $f$ as above, which implies that $\mu=\mu_{0}$ by Theorem 4.3 and Proposition 5.3 . Hence $\mu_{n} \rightarrow \mu$ weakly. Since the converse 
implication is obvious, the first assertion has been shown. The second follows from Proposition 6.4.

Remark 6.6. It is straightforward to see that $x \mapsto \delta_{x}$ is a homeomorphism from $\left(C, d_{T}\right)$ into $\mathcal{P}(C)$ with the weak topology and also an isometry from $\left(C, d_{T}\right)$ into $\left(\mathcal{P}^{1}(C), d_{1}^{W}\right)$. Hence each conclusion of (1) and (2) of Proposition 6.2 implies that the interval $\left[x_{1}, x_{2}\right]$ in $C$ is compact for any $x_{1}, x_{2} \in C$ with $x_{1} \leq x_{2}$. Since $\left(2^{-1} u+C\right) \cap$ $(2 u-C)$ is a non-empty open subset of $\left[2^{-1} u, 2 u\right]$ for any $u \in C$, this forces $E$ to be finite-dimensional. Thus, the finite dimensionality of $E$ is essential in Proposition 6.2. But, there might be a possibility for Propositions 6.3 and 6.4 to hold true beyond the finite-dimensional case.

\section{AGH MEAN INEQUALities}

In this section we consider the Banach space $E=\mathcal{B}(H)$ of bounded operators on a (general) Hilbert space $H$ with the operator norm, and the open cone $C=\mathbb{P}$ consisting of positive invertible operators on $H$. Note that $\mathbb{P}$ is a complete metric space with the Thompson metric $d_{T}$. Let $\Lambda$ be the Karcher barycenter on $\mathcal{P}^{1}(\mathbb{P})$; in particular, for a finitely and uniformly supported measure $\mu=\frac{1}{n} \sum_{j=1}^{n} \delta_{A_{j}}$,

$$
\Lambda_{n}\left(A_{1}, \ldots, A_{n}\right):=\Lambda\left(\frac{1}{n} \sum_{j=1}^{n} \delta_{A_{j}}\right)
$$

is the Karcher or least squares mean of $\left(A_{1}, \ldots, A_{n}\right) \in \mathbb{P}^{n}$, which is uniquely determined by the Karcher equation

$$
\sum_{j=1}^{n} \log \left(X^{-1 / 2} A_{j} X^{-1 / 2}\right)=0
$$

Moreover, $\Lambda: \mathcal{P}^{1}(\mathbb{P}) \rightarrow \mathbb{P}$ is contractive

$$
d_{T}(\Lambda(\mu), \Lambda(\nu)) \leq d_{1}^{W}(\mu, \nu), \quad \mu, \nu \in \mathcal{P}^{1}(\mathbb{P})
$$

See, e.g., [9, 10, 11] for the Karcher equation and Karcher (or Cartan) barycenter.

We consider the complete metric $d_{n}$ on the product space $\mathbb{P}^{n}$

$$
d_{n}\left(\left(A_{1}, \ldots, A_{n}\right),\left(B_{1}, \ldots, B_{n}\right)\right):=\frac{1}{n} \sum_{j=1}^{n} d_{T}\left(A_{j}, B_{j}\right) .
$$


The contraction property of the Karcher barycenter implies that the map

$$
\Lambda_{n}: \mathbb{P}^{n} \rightarrow \mathbb{P}, \quad\left(A_{1}, \ldots, A_{n}\right) \mapsto \Lambda_{n}\left(A_{1}, \ldots, A_{n}\right)
$$

is a Lipschitz map with Lipschitz constant 1.

The arithmetic and harmonic means

$$
\mathcal{A}_{n}\left(A_{1}, \ldots, A_{n}\right)=\frac{1}{n} \sum_{j=1}^{n} A_{j}, \quad \mathcal{H}_{n}\left(A_{1}, \ldots, A_{n}\right)=\left[\frac{1}{n} \sum_{j=1}^{n} A_{j}^{-1}\right]^{-1}
$$

are continuous from $\mathbb{P}^{n}$ to $\mathbb{P}$ and are also Lipschitz with Lipschitz constant 1 for the sup-metric on $\mathbb{P}^{n}$

$$
d_{n}^{\infty}\left(\left(A_{1}, \ldots, A_{n}\right),\left(B_{1}, \ldots, B_{n}\right)\right):=\max _{1 \leq j \leq n} d_{T}\left(A_{j}, B_{j}\right) .
$$

Definition 7.1. For each $n \in \mathbb{N}$ and $\mu_{1}, \ldots, \mu_{n} \in \mathcal{P}(\mathbb{P})$, note that the product measure $\mu_{1} \times \cdots \times \mu_{n}$ is in $\mathcal{P}\left(\mathbb{P}^{n}\right)$. This is easily verified since the support of the product measure is the product of the supports of $\mu_{i}$ 's having the measure 1 . As seen from Proposition 2.1, note also that the push-forward of a $\tau$-additive measure by a continuous map is $\tau$-additive. Hence one can define the following three measures in $\mathcal{P}(\mathbb{P})$, regarded as the geometric, arithmetic and harmonic means of $\mu_{1}, \ldots, \mu_{n}$ :

$$
\begin{aligned}
& \Lambda\left(\mu_{1}, \ldots, \mu_{n}\right):=\left(\Lambda_{n}\right)_{*}\left(\mu_{1} \times \cdots \times \mu_{n}\right), \\
& \mathcal{A}\left(\mu_{1}, \ldots, \mu_{n}\right):=\left(\mathcal{A}_{n}\right)_{*}\left(\mu_{1} \times \cdots \times \mu_{n}\right), \\
& \mathcal{H}\left(\mu_{1}, \ldots, \mu_{n}\right):=\left(\mathcal{H}_{n}\right)_{*}\left(\mu_{1} \times \cdots \times \mu_{n}\right) .
\end{aligned}
$$

Example 7.2. For $\mu=\frac{1}{n} \sum_{j=1}^{n} \delta_{A_{j}}$ and $X \in \mathbb{P}$,

$$
\begin{aligned}
& \Lambda\left(\delta_{X}, \mu\right)=\frac{1}{n} \sum_{j=1}^{n} \delta_{X \# A_{j}}, \\
& \mathcal{A}\left(\delta_{X}, \mu\right)=\frac{1}{n} \sum_{j=1}^{n} \delta_{\left(X+A_{j}\right) / 2}, \\
& \mathcal{H}\left(\delta_{X}, \mu\right)=\frac{1}{n} \sum_{j=1}^{n} \delta_{2\left(X^{-1}+A_{j}^{-1}\right)^{-1}}
\end{aligned}
$$

Proposition 7.3. For every $\mu_{1}, \ldots, \mu_{n} \in \mathcal{P}(\mathbb{P})$,

$$
\mathcal{H}\left(\mu_{1}, \ldots, \mu_{n}\right)=\left[\mathcal{A}\left(\mu_{1}^{-1}, \ldots, \mu_{n}^{-1}\right)\right]^{-1}
$$


where $\mu^{-1}$ is the push-forward of $\mu$ by operator inversion $A \mapsto A^{-1}$.

Proof. For every bounded continuous function $f: \mathbb{P} \rightarrow \mathbb{R}$ we have

$$
\begin{aligned}
\int_{\mathbb{P}} f( & A) d\left[\mathcal{H}\left(\mu_{1}, \ldots, \mu_{n}\right)\right]^{-1}(A) \\
& =\int_{\mathbb{P}} f\left(A^{-1}\right) d \mathcal{H}\left(\mu_{1}, \ldots, \mu_{n}\right)(A) \\
& =\int_{\mathbb{P}^{n}} f\left(\frac{1}{n} \sum_{j=1}^{n} A_{j}^{-1}\right) d\left(\mu_{1} \times \cdots \times \mu_{n}\right)\left(A_{1}, \ldots, A_{n}\right) \\
& =\int_{\mathbb{P}^{n}} f\left(\frac{1}{n} \sum_{j=1}^{n} A_{j}\right) d\left(\mu_{1}^{-1} \times \cdots \times \mu_{n}^{-1}\right)\left(A_{1}, \ldots, A_{n}\right) \\
& =\int_{\mathbb{P}} f(A) d \mathcal{A}\left(\mu_{1}^{-1}, \ldots, \mu_{n}^{-1}\right)(A),
\end{aligned}
$$

which shows that $\left[\mathcal{H}\left(\mu_{1}, \ldots, \mu_{n}\right)\right]^{-1}=\mathcal{A}\left(\mu_{1}^{-1}, \ldots, \mu_{n}^{-1}\right)$.

For a complete metric space $(X, d)$, in addition to $\mathcal{P}^{p}(X)$ with the $p$-Wasserstein metric $d_{p}^{W}$ in (5.1) for $1 \leq p<\infty$, we also consider the set $\mathcal{P}^{\infty}(X)$ of $\mu \in \mathcal{P}(X)$ whose support is a bounded set of $X$, equipped with the $\infty$-Wasserstein metric

$$
d_{\infty}^{W}(\mu, \nu)=\inf _{\pi \in \Pi(\mu, \nu)} \sup \{d(x, y):(x, y) \in \operatorname{supp}(\pi)\}
$$

where $\Pi(\mu, \nu)$ is the set of all couplings for $\mu, \nu$.

Proposition 7.4. For every $p \in[1, \infty]$ and $M=\Lambda, \mathcal{A}, \mathcal{H}$ in (7.6)-(7.8), if $\mu_{1}, \ldots, \mu_{n} \in$ $\mathcal{P}^{p}(\mathbb{P})$ then $M\left(\mu_{1}, \ldots, \mu_{n}\right) \in \mathcal{P}^{p}(\mathbb{P})$. Moreover,

$$
\left(\mu_{1}, \ldots, \mu_{n}\right) \in\left(\mathcal{P}^{p}(\mathbb{P})\right)^{n} \mapsto M\left(\mu_{1}, \ldots, \mu_{n}\right) \in \mathcal{P}^{p}(\mathbb{P})
$$

is Lipschitz continuous with respect to the Wasserstein metric $d_{p}^{W}$.

Proof. Since $\Lambda_{n}: \mathbb{P}^{n} \rightarrow \mathbb{P}$ is a Lipschitz map with Lipschitz constant 1 with respect to $d_{n}$ in (7.4), we can use [11, Lemma 1.3] to see that for each $p \in[1, \infty]$ the push-forward $\operatorname{map}\left(\Lambda_{n}\right)_{*}: \mathcal{P}^{p}\left(\mathbb{P}^{n}\right) \rightarrow \mathcal{P}^{p}(\mathbb{P})$ is Lipschitz with Lipschitz constant 1 with respect to the metric $d_{p}^{W}$, where $d_{p}^{W}$ on $\mathcal{P}^{p}\left(\mathbb{P}^{n}\right)$ is defined in terms of $d_{n}$. Let $\mu_{1}, \ldots, \mu_{n} ; \nu_{1}, \ldots, \nu_{n} \in$ $\mathcal{P}^{p}(\mathbb{P})$. Then it is clear that $\mu_{1} \times \cdots \times \mu_{n} \in \mathcal{P}^{p}\left(\mathbb{P}^{n}\right)$ and hence $\Lambda\left(\mu_{1}, \ldots, \mu_{n}\right)=$ 
$\left(\Lambda_{n}\right)_{*}\left(\mu_{1} \times \cdots \times \mu_{n}\right)$ is in $\mathcal{P}^{p}(\mathbb{P})$. To show the Lipschitz continuity, we may prove more precisely that

$$
\begin{array}{ll}
d_{p}^{W}\left(\Lambda\left(\mu_{1}, \ldots, \mu_{n}\right), \Lambda\left(\nu_{1}, \ldots, \nu_{n}\right)\right) \leq\left[\frac{1}{n} \sum_{j=1}^{n}\left(d_{p}^{W}\left(\mu_{j}, \nu_{j}\right)\right)^{p}\right]^{1 / p} \quad \text { when } 1 \leq p<\infty \\
d_{\infty}^{W}\left(\Lambda\left(\mu_{1}, \ldots, \mu_{n}\right), \Lambda\left(\nu_{1}, \ldots, \nu_{n}\right)\right) \leq \max _{1 \leq j \leq n} d_{\infty}^{W}\left(\mu_{j}, \nu_{j}\right) \quad \text { when } p=\infty .
\end{array}
$$

To prove this, let $\pi_{j} \in \Pi\left(\mu_{j}, \nu_{j}\right), 1 \leq j \leq n$. Since $\pi_{1} \times \cdots \times \pi_{n} \in \Pi\left(\mu_{1} \times \cdots \times\right.$ $\left.\mu_{n}, \nu_{1} \times \cdots \times \nu_{n}\right)$, we have, for the case $1 \leq p<\infty$,

$$
\begin{aligned}
d_{p}^{W} & \left(\left(\Lambda_{n}\right)_{*}\left(\mu_{1} \times \cdots \times \mu_{n}\right),\left(\Lambda_{n}\right)_{*}\left(\nu_{1} \times \cdots \times \nu_{n}\right)\right) \\
& \leq d_{p}^{W}\left(\mu_{1} \times \cdots \times \mu_{n}, \nu_{1} \times \cdots \times \nu_{n}\right) \\
& \leq\left[\int_{\mathbb{P}^{n} \times \mathbb{P}^{n}} d_{n}^{p}\left(\left(A_{1}, \ldots, A_{n}\right),\left(B_{1}, \ldots, B_{n}\right)\right) d\left(\pi_{1} \times \cdots \times \pi_{n}\right)\right]^{1 / p} \\
& =\left[\int_{\mathbb{P}^{n} \times \mathbb{P}_{n}}\left(\frac{1}{n} \sum_{j=1}^{n} d_{T}\left(A_{j}, B_{j}\right)\right)^{p} d\left(\pi_{1} \times \cdots \times \pi_{n}\right)\right]^{1 / p} \\
& \leq\left[\int_{\mathbb{P}^{n} \times \mathbb{P}_{n}} \frac{1}{n} \sum_{j=1}^{n} d_{T}^{p}\left(A_{j}, B_{j}\right) d \pi_{1} \times \cdots \times \pi_{n}\right]^{1 / p} \\
& =\left[\frac{1}{n} \sum_{j=1}^{n} \int_{\mathbb{P} \times \mathbb{P}} d_{T}^{p}\left(A_{j}, B_{j}\right) d \pi_{j}\left(A_{j}, B_{j}\right)\right]^{1 / p} .
\end{aligned}
$$

By taking the infima over $\pi_{j}, 1 \leq j \leq n$, in the last expression, we have the desired $d_{p}^{W}$-inequality when $1 \leq p<\infty$. The proof when $p=\infty$ is similar, so we omit the details.

Since $\mathcal{A}_{n}, \mathcal{H}_{n}: \mathbb{P}^{n} \rightarrow \mathbb{P}$ is Lipschitz with Lipschitz constant 1 with respect to $d_{n}^{\infty}$ in (7.5), we can use [11, Lemma 1.3] again with the metric $d_{p}^{W}$ in terms of $d_{n}^{\infty}$ (in place of $d_{n}$ in the above). For the Lipschitz continuity of $\mathcal{A}\left(\mu_{1}, \ldots, \mu_{n}\right)$ we have, for $1 \leq p<\infty$

$$
\begin{aligned}
& d_{p}^{W}\left(\left(\mathcal{A}_{n}\right)_{*}\left(\mu_{1} \times \cdots \times \mu_{n}\right),\left(\mathcal{A}_{n}\right)_{*}\left(\nu_{1} \times \cdots \times \nu_{n}\right)\right) \\
& \quad \leq d_{p}^{W}\left(\mu_{1} \times \cdots \times \mu_{n}, \nu_{1} \times \cdots \times \nu_{n}\right) \\
& \quad \leq\left[\int_{\mathbb{P}^{n} \times \mathbb{P}^{n}} \max _{1 \leq j \leq n} d_{T}^{p}\left(A_{j}, B_{j}\right) d\left(\pi_{1} \times \cdots \times \pi_{n}\right)\right]^{1 / p}
\end{aligned}
$$




$$
\leq\left[\sum_{j=1}^{n} \int_{\mathbb{P} \times \mathbb{P}} d_{T}^{p}\left(A_{j}, B_{j}\right) d \pi_{j}\left(A_{j}, B_{j}\right)\right]^{1 / p},
$$

which implies that

$$
d_{p}^{W}\left(\mathcal{A}\left(\mu_{1}, \ldots, \mu_{n}\right), \mathcal{A}\left(\nu_{1}, \ldots, \nu_{n}\right)\right) \leq\left[\sum_{j=1}^{n}\left(d_{p}^{W}\left(\mu_{j}, \nu_{j}\right)\right)^{p}\right]^{1 / p} .
$$

For $p=\infty$, we similarly have

$$
d_{\infty}^{W}\left(\mathcal{A}\left(\mu_{1}, \ldots, \mu_{n}\right), \mathcal{A}\left(\nu_{1}, \ldots, \nu_{n}\right)\right) \leq \max _{1 \leq j \leq n} d_{\infty}^{W}\left(\mu_{j}, \nu_{j}\right) .
$$

The proof for $\mathcal{H}\left(\mu_{1}, \ldots, \mu_{n}\right)$ is analogous, or we may use Proposition 7.3 ,

The next theorem is the AGH mean inequalities in the stochastic order for probability measures.

Theorem 7.5. For any $\mu_{1}, \ldots, \mu_{n} \in \mathcal{P}(\mathbb{P})$,

$$
\mathcal{H}\left(\mu_{1}, \ldots, \mu_{n}\right) \leq \Lambda\left(\mu_{1}, \ldots, \mu_{n}\right) \leq \mathcal{A}\left(\mu_{1}, \ldots, \mu_{n}\right)
$$

Proof. Let $f: X \rightarrow \mathbb{R}^{+}$be continuous and monotone. Then by the AGH mean inequalities for operators,

$$
\begin{aligned}
\int_{\mathbb{P}} f d \Lambda\left(\mu_{1}, \ldots, \mu_{n}\right) & =\int_{\mathbb{P}^{n}}\left(f \circ \Lambda_{n}\right)\left(A_{1}, \ldots, A_{n}\right) d\left(\mu_{1} \times \cdots \times \mu_{n}\right)\left(A_{1}, \ldots, A_{n}\right) \\
& \leq \int_{\mathbb{P}^{n}}\left(f \circ \mathcal{A}_{n}\right)\left(A_{1}, \ldots, A_{n}\right) d\left(\mu_{1} \times \cdots \times \mu_{n}\right)\left(A_{1}, \ldots, A_{n}\right) \\
& =\int_{\mathbb{P}} f d \mathcal{A}\left(\mu_{1}, \ldots, \mu_{n}\right),
\end{aligned}
$$

which implies by Proposition 5.3 that $\Lambda\left(\mu_{1}, \ldots, \mu_{n}\right) \leq \mathcal{A}\left(\mu_{1}, \ldots, \mu_{n}\right)$. The proof of $\mathcal{H}\left(\mu_{1}, \ldots, \mu_{n}\right) \leq \Lambda\left(\mu_{1}, \ldots, \mu_{n}\right)$ is similar.

Theorem 7.6. The maps $\Lambda, \mathcal{A}, \mathcal{H}:(\mathcal{P}(\mathbb{P}))^{n} \rightarrow \mathcal{P}(\mathbb{P})$ are monotonically increasing in the sense that if $\mu_{j}, \nu_{j} \in \mathcal{P}(\mathbb{P})$ and $\mu_{j} \leq \nu_{j}$ for $1 \leq j \leq n$, then $M\left(\mu_{1}, \ldots, \mu_{n}\right) \leq$ $M\left(\nu_{1}, \ldots, \nu_{n}\right)$ for $M=\Lambda, \mathcal{A}, \mathcal{H}$.

Proof. Let $f: \mathbb{P} \rightarrow \mathbb{R}^{+}$be a monotone bounded Borel function. We write

$$
\int_{\mathbb{P}} f d \Lambda\left(\mu_{1}, \ldots, \mu_{n}\right)=\int_{\mathbb{P}} g\left(A_{1}\right) d \mu_{1}\left(A_{1}\right),
$$


where

$$
g\left(A_{1}\right):=\int_{\mathbb{P}^{n-1}}\left(f \circ \Lambda_{n}\right)\left(A_{1}, A_{2}, \ldots, A_{n}\right) d\left(\mu_{2} \times \cdots \times \mu_{n}\right)\left(A_{2}, \ldots, A_{n}\right) .
$$

From the monotonicity property of $\Lambda_{n}$, it is immediate to see that $A_{1} \mapsto g\left(A_{1}\right)$ is a monotone bounded Borel function on $\mathbb{P}$. Hence by Proposition 3.6 we have

$$
\int_{\mathbb{P}} f d \Lambda\left(\mu_{1}, \ldots, \mu_{n}\right) \leq \int_{\mathbb{P}} g\left(A_{1}\right) d \nu_{1}\left(A_{1}\right)=\int_{\mathbb{P}} f d \Lambda\left(\nu_{1}, \mu_{2}, \ldots, \mu_{n}\right) .
$$

This implies that $\Lambda\left(\mu_{1}, \mu_{2}, \ldots, \mu_{n}\right) \leq \Lambda\left(\nu_{1}, \mu_{2}, \ldots, \mu_{n}\right)$. Repeating the argument shows that $\Lambda\left(\nu_{1}, \mu_{2}, \ldots, \mu_{n}\right) \leq \Lambda\left(\nu_{1}, \nu_{2}, \mu_{3}, \ldots, \mu_{n}\right)$ and so on. Hence $\Lambda\left(\mu_{1}, \ldots, \mu_{n}\right) \leq$ $\Lambda\left(\nu_{1}, \ldots, \nu_{n}\right)$ follows. The proof is similar for $\mathcal{A}$ and $\mathcal{H}$.

Remark 7.7. One can apply the arguments in this section to other multivariate operator means of $\left(A_{1}, \ldots, A_{n}\right) \in \mathbb{P}^{n}$ having the monotonicity property. For instance, let $P_{t}\left(A_{1}, \ldots, A_{n}\right)$ for $t \in[-1,1]$ be the one-parameter family of multivariate power means interpolating $\mathcal{H}_{n}, \Lambda_{n}, \mathcal{A}_{n}$ as $P_{-1}=\mathcal{H}_{n}, P_{0}=\Lambda_{n}$ and $P_{1}=\mathcal{A}_{n}$. The power mean $P_{t}\left(A_{1}, \ldots, A_{n}\right)$ for $t \in(0,1]$ is defined by the unique positive definite solution of $X=\frac{1}{n} \sum_{j=1}^{n} X \#_{t} A_{j}$, where $A \#_{t} B=A^{1 / 2}\left(A^{-1 / 2} B A^{-1 / 2}\right)^{t} A^{1 / 2}$ denotes the $t$-weighted geometric mean of $A$ and $B$. It is monotonic and Lipschitz

$$
d_{T}\left(P_{t}\left(A_{1}, \ldots, A_{n}\right), P_{t}\left(B_{1}, \ldots, B_{n}\right) \leq \max _{1 \leq j \leq n} d_{T}\left(A_{j}, B_{j}\right) .\right.
$$

Moreover, $P_{t}\left(A_{1}, \ldots, A_{n}\right)$ is monotonically increasing in $t \in[-1,1]$ and

$$
\lim _{t \rightarrow 0} P_{t}\left(A_{1}, \ldots, A_{n}\right)=\Lambda_{n}\left(A_{1}, \ldots, A_{n}\right) .
$$

For power means, see [12] for positive definite matrices and [9, 10] for positive operators on an infinite-dimensional Hilbert space. Then one has the one-parameter family of $P_{t}\left(\mu_{1}, \ldots, \mu_{n}\right)$ for $\mu_{1}, \ldots, \mu_{n} \in \mathcal{P}(\mathbb{P})$ so that each $P_{t}\left(\mu_{1}, \ldots, \mu_{n}\right)$ is monotonically increasing in $\mu_{1}, \ldots, \mu_{n}$ as in Theorem 7.6 and $P_{t}\left(\mu_{1}, \ldots, \mu_{n}\right)$ is monotonically increasing in $t$, extending the AGH mean inequalities in Theorem 7.5. Moreover,

$$
P_{s}\left(\mu_{1}, \ldots, \mu_{n}\right) \leq \Lambda\left(\mu_{1}, \ldots, \mu_{n}\right) \leq P_{t}\left(\mu_{1}, \ldots, \mu_{n}\right)
$$

for $-1 \leq s<0<t \leq 1$ as in Theorem 7.5.

Now assume that $\mathbb{P}$ is the cone of positive definite matrices of some fixed dimension, and let $\mu_{j} \in \mathcal{P}^{1}(\mathbb{P}), 1 \leq j \leq n$. For any continuous bounded and monotone $f: \mathbb{P} \rightarrow$ 
$\mathbb{R}^{+}$we see by (17.10) that

$$
\int_{\mathbb{P}} f d P_{t}\left(\mu_{1}, \ldots, \mu_{n}\right)=\int_{\mathbb{P}^{n}}\left(f \circ P_{t}\right)\left(A_{1}, \ldots, A_{n}\right) d\left(\mu_{1} \times \cdots \times \mu_{n}\right)\left(A_{1}, \ldots, A_{n}\right)
$$

increases as $t \nearrow 0$ and decreases as $t \searrow 0$ to

$$
\int_{\mathbb{P}^{n}}\left(f \circ \Lambda_{n}\right)\left(A_{1}, \ldots, A_{n}\right) d\left(\mu_{1} \times \cdots \times \mu_{n}\right)\left(A_{1}, \ldots, A_{n}\right)=\int_{\mathbb{P}} f d \Lambda\left(\mu_{1}, \ldots, \mu_{n}\right) .
$$

Hence by Corollary 6.5,

$$
\lim _{t \rightarrow 0} d_{1}^{W}\left(P_{t}\left(\mu_{1}, \ldots, \mu_{n}\right), \Lambda\left(\mu_{1}, \ldots, \mu_{n}\right)\right)=0
$$

It would be interesting to know whether this convergence holds true in the infinitedimensional case as well.

Remark 7.8. Several issues arise related to $\Lambda\left(\mu_{1}, \ldots, \mu_{n}\right)$. For example, it is interesting to consider existence and uniqueness for the least squares mean on $\mathcal{P}^{1}(\mathbb{P})$;

$$
\underset{\mu \in \mathcal{P}^{1}(\mathbb{P})}{\arg \min } \sum_{j=1}^{n} d_{1}^{W}\left(\mu, \mu_{j}\right)^{2}
$$

and a connection with the probability measure $\Lambda\left(\mu_{1}, \ldots, \mu_{n}\right)$. Moreover, the probability Borel measure equation

$$
x=\mathcal{A}\left(x \#_{t} \mu_{1}, \ldots, x \#_{t} \mu_{n}\right), \quad \mu_{j} \in \mathcal{P}_{c p}(\mathbb{P}), t \in(0,1],
$$

where $\mu \#{ }_{t} \nu=f_{*}(\mu \times \nu)$ is the push-forward by the $t$-weighted geometric mean map $f(A, B)=A \#{ }_{t} B$, seems to have a unique solution in $\mathcal{P}_{c p}(\mathbb{P})$, the set of probability measures with compact support.

\section{Acknowledgements}

The work of F. Hiai was supported in part by Grant-in-Aid for Scientific Research (C)17K05266. The work of Y. Lim was supported by the National Research Foundation of Korea (NRF) grant funded by the Korea government (MEST) No.NRF2015R1A3A2031159. 


\section{REFERENCES}

[1] P. Billingsley, Convergence of Probability Measures, Second edition, A Wiley-Interscience Publication, John Wiley \& Sons, New York, 1999.

[2] K. Deimling, Nonlinear Functional Analysis, Springer Verlag, Berlin, 1985.

[3] R. M. Dudley, Real Analysis and Probability, The Wadsworth \& Brooks/Cole Mathematics Series. Wadsworth \& Brooks/Cole Advanced Books \& Software, Pacific Grove, CA, 1989.

[4] D. H. Fremlin, Measure Theory, Volumes 1-5, Lulu.com, 2000.

[5] G. Gierz, K. Hofmann, K. Keimel, J. Lawson, M. Mislove, and D. Scott, Continuous Lattices and Domains, Cambridge University Press, 2003.

[6] S. Kim, J. Lawson and Y. Lim, Barycentric maps for compactly supported measures, submitted.

[7] J. Lawson, Ordered probability spaces, J. Math. Anal. Appl. 455 (2017), 167-179.

[8] J. Lawson and Y. Lim, A Lipschitz constant formula for vector addition in cones with applications to Stein-like equations, Positivity 16 (2012), 81-95.

[9] J. Lawson and Y. Lim, Weighted means and Karcher equations of positive definite operators, Proc. Natl. Acad. Sci. USA. 110 (2013), 15626-15632.

[10] J. Lawson and Y. Lim, Karcher means and Karcher equations of positive operators, Trans. Amer. Math. Soc. Series B 1 (2014), 1-22.

[11] J. Lawson and Y. Lim, Contractive barycentric maps, J. Operator Theory 77 (2017), 87-107.

[12] Y. Lim and M. Pálfia, Matrix power means and the Karcher mean, J. Funct. Anal. 262 (2012), $1498-1514$.

[13] K.-T. Sturm, Probability measures on metric spaces of nonpositive curvature, in Heat Kernels and Analysis on Manifolds, Graphs, and Metric Spaces (Paris, 2002), pp. 357-390, Contemp. Math., 338, Amer. Math. Soc., Providence, RI, 2003.

[14] A. C. Thompson, On certain contraction mappings in a partially ordered vector space, Proc. Amer. Math. Soc. 14 (1963), 438-443.

[15] C. Villani, Topics in Optimal Transportation, Graduate Studies in Mathematics, Vol. 58, Amer. Math. Soc., Providence, RI, 2003.

Tohoku University (Emeritus), Hakusan 3-8-16-303, Abiko 270-1154, Japan

E-mail address: hiai.fumio@gmail.com

Department of Mathematics, Louisiana State University, Baton Rouge, LA70803, USA

E-mail address: lawson@math.1su.edu

Department of Mathematics, Sungkyunkwan University, Suwon 440-746, Korea

E-mail address: ylim@skku.edu 